\title{
Situação nutricional de crianças em município de privilegiado Índice de Desenvolvimento Humano do semiárido brasileiro e sua relação com Insegurança Alimentar
}

\author{
Élida Mara Braga Rocha ${ }^{1}$, Roberto Teixeira de Lima ${ }^{2}$, Derlange Belizário Diniz ${ }^{3}$, \\ Paulo César de Almeida ${ }^{4}$
}

\begin{abstract}
As situações de Insegurança Alimentar (IA) podem ser detectadas a partir de diferentes problemas desde fome até obesidade. A análise da Segurança Alimentar e Nutricional por meio de estudos populacionais vem ocupando de forma crescente a agenda pública do Brasil. O objetivo desta pesquisa foi correlacionar o perfil nutricional com a situação de IA em crianças menores de cinco anos de idade em município de privilegiado Índice de Desenvolvimento Humano do semiárido brasileiro, Crato (CE). A pesquisa foi do tipo transversal, probabilística, com seleção das crianças por amostragem sistemática no "Dia D" da Campanha Nacional de Vacinação, em agosto de 2010. Um questionário socioeconômico e a Escala Brasileira de Insegurança Alimentar (EBIA) foram aplicados, além da aferição de peso e altura para a associação entre situação nutricional e situação de IA por meio do teste Qui-Quadrado $(\not<0,05)$. Foram coletados dados de 352 crianças. Na análise da situação nutricional, a prevalência de sobrepeso foi acentuada para o indicador peso para altura $(16,8 \%)$. Entretanto, quanto ao índice altura para idade, obteve-se uma prevalência elevada para desnutrição $(7,1 \%)$. Contudo, não houve associação de desnutrição nem de sobrepeso com IA, assim, a EBIA se mostrou como indicador não relacionado aos distúrbios nutricionais.
\end{abstract}

Palavras-chave: Segurança Alimentar e Nutricional, sobrepeso, crianças.

\section{Nutritional status of children in the city of privileged Human Development Index of Brazilian semi-arid and its relation to food insecurity}

Food Insecurity (FI) situation may be detected from different problems from hunger to obesity. The analysis of Food Security through population-based studies is increasingly occupying the public agenda in Brazil. The objective of this research was to correlate the nutritional profile with the FI situation in children under five years old in the city of privileged Human Development Index of a Brazilian semi-arid (Crato-CE). The research was cross-sectional and probabilistic, with the selection of children by systematic sampling in the "D Day" of the National Vaccination Campaign, in August 2010. A socioeconomic questionnaire and the Brazilian Food Insecurity Scale (EBIA) were applied, along with the measurement of height and weight for the association between nutritional status and situation of FI by using the chi-square $(\phi<0.05)$. Data were collected from 352

\footnotetext{
${ }^{1}$ Mestre em Ciências da Nutrição pela Universidade Federal da Paraíba (UFPB). Correspondência: Av. Engenheiro Heitor Antonio Eiras Garcia, 396. Apto 81, Bloco México. Jardim Esmeralda. São Paulo, SP. CEP 05588-001. Tel. (11)4301-7086/(11)97239-7554/(11)95342-9021. E-mail: elidamara_92@yahoo.com.br.

${ }^{2}$ Doutor em Saúde Pública pela Faculdade de Saúde Pública, Universidade de São Paulo (USP). Prof. Adjunto do Departamento de Nutrição da UFPB.

${ }^{3}$ Doutora em Ciências da Nutrição pela Universidade Estadual de Campinas (UNICAMP). Profa Adjunta do Centro de Ciências da Saúde, Universidade Estadual do Ceará (UECE).

${ }^{4}$ Doutor em Saúde Pública pela Faculdade de Saúde Pública, USP. Prof. Adjunto do Centro de Ciências da Saúde, UECE.
} 
children. In the analysis of nutritional situation, the prevalence of overweight was significant to the weight to stature indicator $(16.8 \%)$. However, in relation to the stature to age indicator, it was obtained a prevalence to malnutrition (7.1\%). Moreover, there was neither malnutrition nor overweight association with FI, demonstrating, therefore, that EBIA has revealed as an indicator not related to nutrition desorders.

Key-words: Food and Nutrition Security, overweight, children.

\section{INTRODUÇÃO}

No Brasil, a situação de Segurança Alimentar e Nutricional (SAN) é a realização do direito humano a uma alimentação saudável, acessível, de qualidade, em quantidade suficiente, de modo permanente, sem comprometer o acesso a outras necessidades essenciais, com base em práticas alimentares saudáveis, respeitando as diversidades culturais e sendo sustentável do ponto de vista socioeconômico e agroecológico ${ }^{[1]}$.

As situações de insegurança alimentar e nutricional podem ser detectadas a partir de diferentes tipos de problemas, tais como: fome, obesidade, doenças associadas à má alimentação, consumo de alimentos de qualidade duvidosa ou prejudicial à saúde, estrutura de produção de alimentos predatória em relação ao ambiente e bens essenciais com preços abusivos e imposição de padrões alimentares que não respeitem a diversidade cultural local [2].

Diante disso, a SAN tornou-se tema de discussão de âmbito mundial. Além do intenso e amplo debate pela comunidade científica, é necessário consolidar e dinamizar a produção de conhecimento sobre o tema, contribuindo para o desenvolvimento e implementação de ações e políticas de SAN [3,4].

A pesquisa nacional verificou que 65,6 milhões de brasileiros sofrem com algum grau de Insegurança Alimentar (IA) [5. Consequentemente, a análise e o monitoramento da SAN por meio de estudos populacionais vêm ocupando de forma crescente a agenda pública do Brasil, devido ao seu papel como marcador de desigualdades inerentes ao sistema econômico vigente [ø.

Neste contexto, observa-se na Região do Semiárido brasileiro um dos mais sérios quadros de miséria do país, sendo a desnutrição infantil um problema ainda presente na primeira década do século XXI [7]. Muito embora a transição nutricional, caracterizada pela coexistência de baixo peso e sobrepeso na população seja uma realidade brasileira, é alarmante o aumento progressivo da prevalência de sobrepeso/obesidade ${ }^{[8]}$.

A Pesquisa de Orçamentos Familiares (POF) 2008-2009, divulgada em 2010, confirma que o crescimento do excesso de peso em crianças, adolescentes e adultos brasileiros ultrapassa o padrão internacional [?].

O que se observa é que nos países em desenvolvimento e mais urbanizados, a população tem uma tendência de padrão de vida semelhante, com consumo de alimentos altamente calóricos e estilo de vida sedentário, resultando em acúmulo de gordura corporal devido à desproporcional relação consumo e gasto de energia [10-12]. As graves consequências do sobrepeso e obesidade na infância, as dificuldades para seu tratamento e o custo elevado para a sociedade, com o aumento das morbimortalidades, fazem deste agravo um problema de Saúde Pública relevante.

Existem cinco métodos utilizados para mensuração e avaliação de IA: o método da Food and Agriculture Organization (FAO) para estimar as calorias disponíveis per capita, pesquisas de renda e gasto domiciliar, pesquisas do consumo de alimentos no nível individual, antropometria e a escala de experiência de IA no domicílio [13].

Os dois métodos mais bem aceitos em pesquisas transversais são a antropometria e a escala de percepção de IA, pela simplicidade na coleta, processamento e análise de dados, além de ser de baixo custo e rápida aplicação. A antropometria, definida como medição direta da estatura e da altura, representa uma maneira racional e eficiente para a coleta de dados sobre a situação nutricional da população em estudos epidemiológicos, sendo, entretanto, um método indireto para medir a IA [14]. Já a escala de percepção de IA é utilizada na mensuração direta do fenômeno, que leva em consideração uma investigação qualitativa e 
quantitativa, permitindo capturar não apenas as dimensões físicas, mas também psicológicas [15].

Além disso, Vasconcelos [16] e Teixeira-Lima [17] citam outros indicadores para mensurar as nuances das condições de vida da população, entre os quais o de maior destaque é o Índice de Desenvolvimento Humano (IDH), sendo este acompanhado pelo Programa das Nações Unidas para o Desenvolvimento (PNUD). Assim, desde 1993, o PNUD utiliza o IDH em seu relatório anual por ser uma maneira padronizada de avaliação e medida do bem-estar de uma população [18].

Diante disso surgem algumas questões. Qual a situação nutricional no grupo mais vulnerável - a população infantil - em município de privilegiado IDH? Existe uma situação nutricional determinante para indivíduos em situação de IA? A antropometria e a escala de segurança alimentar medem de forma similar o mesmo fenômeno? A nosso ver, estas questões não são de fácil resposta, já que esta análise não é possível de ser elaborada sob um único foco de fator causal, visto que implica em um processo multifacetado.

Neste sentido, buscando maior compreensão sobre essa questão, o objetivo desta pesquisa foi correlacionar o perfil nutricional com a situação de IA de crianças menores de cinco anos de idade no município de Crato, um dos melhores IDH do Ceará, além de caracterizar e avaliar a situação nutricional dessas crianças.

\section{METODOLOGIA}

A pesquisa foi do tipo transversal, de abordagem descritiva e analítica, de base populacional do município de Crato (CE).

O município investigado está inserido na Região do Semiárido brasileiro, no Estado do Ceará, possuindo uma área de $1.157,91 \mathrm{~km}^{2}$ e uma população de 121.462 habitantes, de acordo com dados de 2010 do Instituto Brasileiro de Geografia e Estatística (IBGE) [19].

O município de Crato é um dos 25 pertencentes à Mesorregião do Sul Cearense que representa um polo dos mais importantes sob os aspectos ambiental, socioeconômico e cultural do Estado. Quanto às características ambientais, as temperaturas médias anuais variam entre 23 e $27^{\circ} \mathrm{C}$ (mínima e máxima), com precipitação média anual de $1.090,90 \mathrm{~mm}$. As coberturas vegetais encontradas são representadas, principalmente, por matas ciliares, caatingas, mata seca, mata úmida e cerrado [20].

Em 2000, o IDH-Municipal (IDHM) de Crato, utilizado para aferir o nível de desenvolvimento humano em municípios a partir das variáveis educação, longevidade e renda, era de 0,716, considerado de médio desenvolvimento humano. Em comparação aos outros municípios do Estado, Crato apresenta a 5a melhor posição, atrás apenas da capital do Estado e de três municípios da Região Metropolitana de Fortaleza [18].

Para cálculo da amostra, a população do estudo foi de 10.500 crianças menores de cinco anos de idade que foram imunizadas no município, segundo os dados da primeira etapa da Campanha Nacional de Vacinação, referente ao ano de 2009 [21]. Foi considerada a prevalência de $67,5 \%$ de IA para crianças menores de cinco anos de idade no Nordeste, segundo a Pesquisa Nacional por Amostra de Domicílios (PNAD-2004) [22]. Aplicou-se um erro amostral de 5\% com intervalos de confiança de $95 \%$ e acréscimo de $10 \%$ para perda amostral, totalizando uma amostra de 360 crianças.

A amostragem foi do tipo probabilística, com o sorteio aleatório de seis dos trinta Postos de Vacinação, mantendo-se a proporcionalidade dos conglomerados rurais (2) e urbanos (4). Em seguida, a amostra foi estratificada por área rural $(n=90)$ e urbana $(n=270)$. No dia 14 de agosto de 2010 (segunda etapa de vacinação), as crianças foram selecionadas por ordem de chegada, com definição prévia do intervalo amostral, segundo a demanda do posto de vacinação na primeira etapa da campanha.

Foi utilizado um questionário pré-codificado direcionado aos responsáveis das crianças selecionadas, abordando informações sobre condições socioeconômicas, tais como classe socioeconômica, escolaridade em anos completos concluídos do chefe da família, participação em programas sociais, gênero e idade da criança e cor autorreferida do chefe da família.

Sobre a classe social das famílias, foi utilizada a classificação de acordo com critérios estabelecidos pela Associação Brasileira de Empresas de Pesquisas (ABEP) [23] que se baseia na pontuação total que uma família alcança em uma escala de nove itens (tipo e 
número de bens materiais e serviços disponíveis no domicílio) somados à escolaridade do chefe da família, construída de modo a maximizar a correlação da pontuação total com a renda familiar. A classificação ABEP prevê originalmente oito classes decrescentes de poder aquisitivo (A1, A2, B1, B2, C1, C2, D e E) que respeitam a pontuação total dos itens dos domicilios, podendo variar de 0 a 46 pontos. Para o presente trabalho, as classes socioeconômicas foram agrupadas em A-B, C, D e E, devido à reduzida frequência nas classes A, B e C.

A avaliação da situação nutricional foi implementada mediante análise antropométrica. Foram utilizadas medidas de peso e altura, segundo as normas técnicas da Organização Mundial de Saúde, adotadas pelo Ministério da Saúde [24], em que os menores de dois anos foram pesados despidos e os maiores de dois anos com uma peça de roupa íntima (calcinha/cueca). Tais medidas, tomadas em duplicata, foram realizadas por uma dupla de antropometristas previamente treinada.

Os instrumentos utilizados para mensuração de peso e altura das crianças menores de dois anos foram balanças pediátricas digitais Welmy ${ }^{\circledR}$ com capacidade de $16 \mathrm{~kg}$ e intervalos de $10 \mathrm{~g}$. Para aferição do comprimento, foi usado infantômetro de madeira portátil com fita métrica de $100 \mathrm{~cm}$ e escala de $1 \mathrm{~mm}$. Já para crianças maiores de dois anos, foram utilizadas as balanças da marca Tanita ${ }^{\circledR}$ com capacidade de 150 $\mathrm{kg}$ e intervalos de $100 \mathrm{~g}$ e, para aferição da estatura, foi utilizado o antropômetro Alturexata ${ }^{\circledR}$ de leitura lateral apoiado na parede, com extensão de 0 a 2 metros e graduação em milímetros.

Para a análise da situação nutricional, foram utilizados os índices Peso/Idade (P/I), Peso/Altura (P/A), Índice de Massa Corporal/Idade (IMC/I) e Altura/Idade (A/I). Esses índices, expressos em escores $Z$, foram calculados por meio de um padrão de referência que reproduz a distribuição dos índices em ótimas condições de alimentação e saúde da criança. Para tanto foi utilizado o software ANTRHO [25], no qual, para a determinação de agravos nutricionais, utiliza os extremos $\leq-2$ escores $Z$ para desnutrição e $\geq$ +2 escores $Z$ para sobrepeso/obesidade infantil. Para eutrofia ou "normalidade" foi considerado o intervalo de escores $Z$ de $>-2$ a $<+2$, de acordo com World Health Organization (WHO) ${ }^{[26] . ~}$
Já para o diagnóstico da situação de segurança alimentar, foi utilizada a Escala Brasileira de Insegurança Alimentar (EBIA), que classifica as famílias em uma das quatro categorias, segundo os escores de pontuação. Cada resposta afirmativa do questionário de IA representa 1 ponto, sendo a pontuação da escala sua soma, variando em uma amplitude de 0 a 15 pontos; sendo a segurança 0 pontos; insegurança leve 1-5; insegurança moderada 6-10 pontos; e insegurança grave 11-15 pontos em famílias com menores de 18 anos [27].

As análises estatísticas foram realizadas com auxilio do software Statistical Packeage for the Social Sciences (SPSS) 14.0 for Windows. Tabelas e gráficos foram construídos para apresentação dos resultados. Primeiro, realizou-se à análise descritiva das informações que caracterizam a população estudada. Segundo, foi examinada a associação existente entre os indicadores antropométricos e a situação de IA por meio de teste de Qui-Quadrado $\left(\chi^{2}\right)$, fixado $p<0,05$. Foi realizada análise bivariada, calculadas as Razões de Chances brutas (RCb), com intervalos de confiança (IC95\%) e respectivos valores de $p$.

Quanto aos aspectos éticos, a pesquisa foi previamente submetida à análise pelo Comitê de Ética em Pesquisa do Hospital Universitário Lauro Wanderly (CEP/HULW), da Universidade Federal da Paraíba, onde obteve aprovação para sua realização e publicação sob protocolo de no 346/10, segundo as recomendações da Resolução 196/96 [28].

\section{RESULTADOS}

Foram aplicados 370 questionários às famílias de crianças menores de cinco anos. Contudo houve perda de 18 questionários por falta de informações essenciais para antropometria como data de nascimento, peso ou altura da criança, correspondendo a uma perda amostral dentro do esperado, menor que $10 \%$.

Foi obtida proporcionalidade da área urbana e rural, já que aproximadamente $25 \%$ das 352 crianças pertenciam à área rural (conforme registrado na Tabela 1). A proporção de crianças do gênero masculino $(51,4 \%)$ esteve muito próxima à do gênero feminino (48,3\%), sendo 56,8\% com mais de 24 meses de idade. Sobre o chefe da família, a maioria se auto-declarou de cor não branca $(74,1 \%)$ e com mais de nove anos de estudo (46\%). 
Segundo o Critério de Classificação Econômica Brasil [23], os níveis socioeconômicos mais representativos foram das classes $\mathrm{C}$ e $\mathrm{D}$, abrangendo aproximadamente $3 / 4$ das famílias $(75,1 \%)$. Vale destacar que $14,7 \%$ das famílias eram da classe socioeconômica $\mathrm{A}$ ou $\mathrm{B}$, demonstrando a diversificação da amostra. Sendo observado que 40,2\% das famílias apresentavam renda per capita menor que $1 / 4$ do salário mínimo, correspondendo a menos de $\mathrm{R} \$ 127,5$ por pessoa, corroborando com os $48 \%$ das famílias que recebiam benefício social, conforme Tabela 1 .

Tabela 1. Caracterização das famílias de crianças de zero a 59 meses de idade, segundo as condições socioeconômicas. Crato (CE), Brasil, 2010

\begin{tabular}{|c|c|c|}
\hline Variáveis & Número & $\begin{array}{c}\text { Proporção } \\
(\%)\end{array}$ \\
\hline \multicolumn{3}{|l|}{ Área } \\
\hline Urbano & 268 & 76,1 \\
\hline Rural & 84 & 23,9 \\
\hline \multicolumn{3}{|l|}{ Sexo da Criança } \\
\hline Masculino & 181 & 51,4 \\
\hline Feminino & 171 & 48,6 \\
\hline \multicolumn{3}{|l|}{ Idade das crianças (meses) } \\
\hline$<6$ & 45 & 12,8 \\
\hline $6-11$ & 41 & 11,6 \\
\hline $12-23$ & 66 & 18,8 \\
\hline $24-35$ & 75 & 21,3 \\
\hline $36-47$ & 64 & 18,2 \\
\hline $48-59$ & 61 & 17,3 \\
\hline \multicolumn{3}{|l|}{ Cor/raça do Chefe da Família } \\
\hline Branca & 91 & 25,9 \\
\hline Não Branca & 261 & 74,1 \\
\hline \multicolumn{3}{|l|}{ Escolaridade do Chefe da Família } \\
\hline$\leq 4$ anos de estudo & 88 & 25,4 \\
\hline $5-8$ anos de estudo & 99 & 28,6 \\
\hline$\geq 9$ anos de estudo & 159 & 46,0 \\
\hline \multicolumn{3}{|l|}{ Classe Socioeconômica } \\
\hline $\mathrm{A}-\mathrm{B}$ & 51 & 14,7 \\
\hline $\mathrm{C}$ & 152 & 43,9 \\
\hline $\mathrm{D}$ & 108 & 31,2 \\
\hline $\mathrm{E}$ & 35 & 10,1 \\
\hline \multicolumn{3}{|l|}{ Recebimento de Benefício Social } \\
\hline $\operatorname{Sim}$ & 169 & 48,0 \\
\hline Não & 183 & 52,0 \\
\hline
\end{tabular}


De acordo com a Figura, quando aplicada a EBIA, 42,6\% das famílias de crianças menores de cinco anos de idade encontravam-se em situação de segurança alimentar, sendo os níveis de IA moderada ou grave, quantidade inadequada e/ou fome, encontrada em aproximadamente $1 / 4$ das famílias $(25,6 \%)$.

Figura. Prevalência de Insegurança Alimentar (IA) nas famílias de crianças de zero a 59 meses de idade do município de Crato (CE), Brasil, 2010

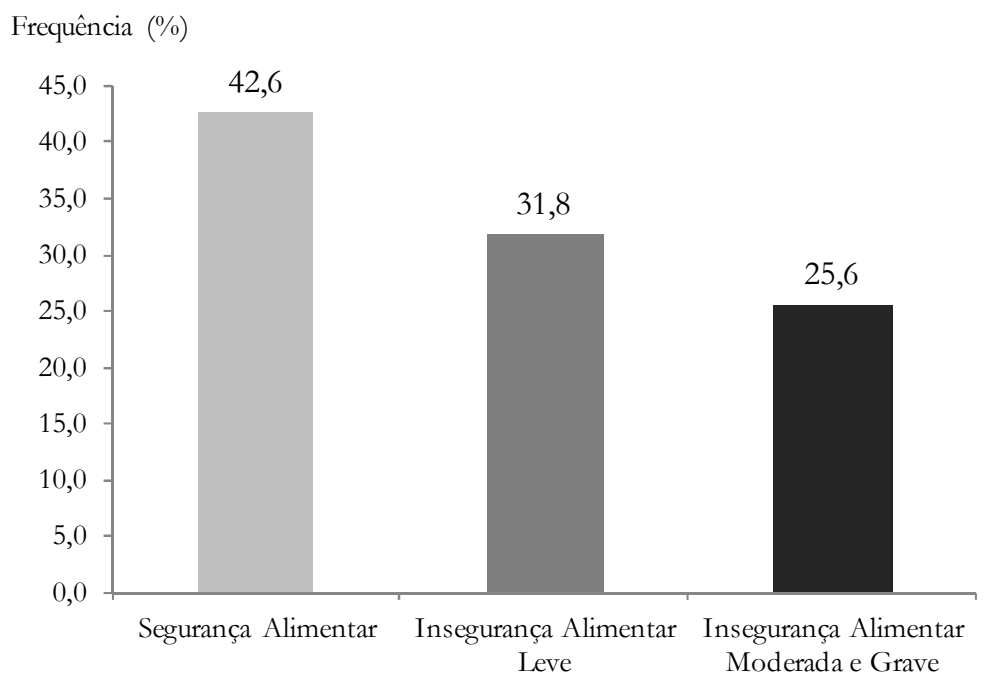

No tocante à situação nutricional, a prevalência de eutrofia variou entre $82 \%$ e $89 \%$ para todos os indicadores antropométricos. Verificou-se uma frequência muito baixa de desnutrição quando foram adotados os indicadores $\mathrm{P} / \mathrm{I}, \mathrm{P} / \mathrm{A}$ e IMC/I, correspondendo a $1,4 \%, 1,1 \%$ e $0,9 \%$ das crianças, respectivamente. Em contraste, considerando os mesmos indicadores, o sobrepeso foi acentuado, 9,9\%, $16,8 \%$ e $16,2 \%$. No que diz respeito ao índice A/I, obteve-se uma prevalência elevada para déficit estatural (7,1\%), conforme mostrado na Tabela 2.

Quando analisada a relação de SAN com a nutrição infantil, mesmo que não tenha sido confirmada associação estatisticamente significativa $(p$ $>0,05)$, conforme mostrado na Tabela 2 , observa-se a presença de crianças com sobrepeso/obesidade em famílias em situação de IA grave, de acordo com os indicadores P/I (5,7\%), P/A (11,9\%) e IMC/I (12,3\%). Já para crianças classificadas como desnutridas, pelo indicador antropométrico A/I, 12\% delas apresentaram frequência de IA grave, demonstrando diferenças entre as prevalências de IA quando se analisa a SAN por meio de diferentes indicadores antropométricos.

\section{DISCUSSÃO}

\section{Déficit nutricional}

A situação nutricional identificada pelos valores dos indicadores P/I, P/A e IMC (1,4\%, 1,1\% e $0,9 \%$, respectivamente) demonstram valores próximos aos encontrados na distribuição de referência, esperados em populações ideais [26], semelhante aos achados pela Pesquisa Nacional de Demografia e Saúde da Criança e da Mulher (PNDS-2006), tanto para o Brasil $(1,8 \%$ e $1,5 \%)$ quanto para o Nordeste $(2,2 \%$ e $1,7 \%$ ), indicando, assim, virtual equilíbrio da situação de desnutrição aguda [29].

De modo geral, as pesquisas nacionais nos últimos anos vêm apontando um considerável declínio da desnutrição, indicando que em pouco mais de dez anos a desnutrição infantil poderá deixar de ser um problema de Saúde Pública no Brasil. Os principais 
fatores destacados para tal evolução se devem ao aumento da escolaridade materna, ao crescimento do poder aquisitivo das famílias, à expansão da assistência à saúde e à melhoria das condições de saneamento [30].
Embora a condição de desnutrição infantil venha sendo minimizada nos últimos anos, a situação de IA ainda se faz presente no contexto dessas famílias.

Tabela 2. Caracterização da amostra, situação de segurança alimentar e razões de chance para insegurança alimentar, de acordo com o estado nutricional infantil. Crato (CE), Brasil, 2010

\begin{tabular}{|c|c|c|c|c|c|c|c|c|c|c|}
\hline \multirow{3}{*}{$\begin{array}{l}\text { Variáveis } \\
\text { (escores } Z)\end{array}$} & \multirow{2}{*}{\multicolumn{2}{|c|}{ Geral }} & \multirow{3}{*}{$\begin{array}{l}\text { SA } \\
\%\end{array}$} & \multicolumn{3}{|c|}{ Insegurança Alimentar } & \multirow{3}{*}{$\begin{array}{c}\chi^{2} \\
(p \text {-valor })\end{array}$} & \multirow{2}{*}{\multicolumn{3}{|c|}{ SA versus IA }} \\
\hline & & & & \multirow{2}{*}{$\begin{array}{l}\text { Leve } \\
\qquad \%\end{array}$} & \multirow{2}{*}{$\begin{array}{c}\text { Moderada } \\
0 \%\end{array}$} & \multirow{2}{*}{$\begin{array}{c}\text { Grave } \\
\%\end{array}$} & & & & \\
\hline & $n$ & $\%$ & & & & & & $\mathbf{R C b}$ & IC $_{95 \%}$ & $p^{*}$ \\
\hline \multicolumn{7}{|l|}{ Peso/Idade } & \multicolumn{4}{|l|}{0,221} \\
\hline$\leq-2$ & 5 & 1,4 & 20 & 20 & 60 & 0 & & 2,86 & $0,32-3,86$ & 0,329 \\
\hline$\geq+2$ & 35 & 9,9 & 54,3 & 28,6 & 11,4 & 5,7 & & 0,6 & $0,30-1,21$ & 0,153 \\
\hline \multicolumn{11}{|l|}{$>-2 a<$} \\
\hline+2 & 312 & 88,6 & 41,6 & 32,4 & 18,6 & 7,4 & & 1 & & \\
\hline
\end{tabular}

\section{Peso/Altura}

$\begin{array}{lcccccr}\leq-2 & 4 & 1,1 & 50 & 25 & 25 & 0 \\ \geq+2 & 59 & 16,8 & 42,4 & 28,8 & 16,9 & 11, \\ >-2 \mathrm{a}< & & & & & & \\ +2 & 289 & 82,1 & 42,6 & 32,5 & 18,7 & 6,2\end{array}$

\section{IMC/Idade}

$\begin{array}{lcccccc}\leq-2 & 3 & 0,9 & 33,3 & 33,3 & 33,3 & 0 \\ \geq+2 & 57 & 16,2 & 40,3 & 31,6 & 15,8 & 12,3 \\ >-2 a< & & & & & & \\ +2 & 292 & 83 & 43,2 & 31,8 & 18,8 & 6,2\end{array}$

\section{Altura/Idade}

$\begin{array}{lcccccc}\leq-2 & 25 & 7,1 & 32 & 24 & 32 & 12 \\ \geq+2 & 13 & 3,7 & 38,5 & 38,5 & 15,3 & 7,7 \\ >-2 \mathrm{a}< & & & & & & \\ +2 & 314 & 89,2 & 43,6 & 32,2 & 17,5 & 6,7\end{array}$

0,812

$$
\begin{array}{ccc}
0,74 & 0,10-5,33 & 0,765 \\
1,01 & 0,57-1,78 & 0,979 \\
& & \\
1 & &
\end{array}
$$

\begin{tabular}{|c|c|c|}
\hline ) & 1,52 & $0,14-16,93$ \\
\hline & 1,12 & $0,63-2,0$ \\
\hline
\end{tabular}

0,747

0,532

$\begin{array}{cccc}12 & 1,65 & 0,69-3,92 & 0,258 \\ , 7 & 1,24 & 0,40-3,87 & 0,713 \\ & & & \\ 6,7 & 1 & & \end{array}$

$\mathrm{SA}=$ Segurança Alimentar; IA = Insegurança Alimentar; $\chi^{2}=$ Qui-quadrado; RCb = Razão de Chance bruto; IC = Intervalo de Confiança; IMC = Índice de Massa Corporal; $p^{*}$ calculado para RC's das tabelas de associação. 
Em contraponto, neste estudo, 7,1\% das crianças investigadas passaram por algum momento de desnutrição, segundo o indicador $\mathrm{A} / \mathrm{I}$, alterando seu padrão de crescimento estatural. Essa prevalência foi ainda superior à encontrada, em 2005, para os municípios do semiárido como um todo $(6,6 \%)^{[31]}$, em pesquisas nacionais de 2006 e 2008/09, para a Região do Nordeste (5,8\% e 5,9\%, respectivamente), como também para o Brasil $(6,7 \%$ e $6,0 \%)$, segundo a PNDS-2006 [29] e POF 2008/09 [9], respectivamente. Entretanto, é inferior ao encontrado no Semiárido do Estado do Ceará (8,6\%), na Chamada Nutricional de 2005 [32].

Ao analisar o perfil nutricional infantil descrito neste estudo, pode-se observar semelhança com as pesquisas realizadas em 2009 e 2010 em municípios do semiárido paraibano e pernambucano, onde em ambas as situações foram constatadas prevalências baixas para déficit de peso e elevadas para déficit estatural [33,34].

Em localidades em que a transição nutricional ainda se apresenta em suas formas iniciais, como Bogotá, na Colômbia, escolares em IA apresentaram três vezes mais chances de estar abaixo do peso do que as crianças em segurança alimentar, para o índice $\mathrm{P} / \mathrm{I}\left(\mathrm{IC}_{95 \%}: 1,6-5,4 ; p=0,0007\right)$. Contudo, para os indicadores $\mathrm{P} / \mathrm{A}$ ou $\mathrm{A} / \mathrm{I}$ não foram observadas relações com IA tanto para desnutrição como sobrepeso infantil [35].

Vale salientar que o índice $\mathrm{P} / \mathrm{I}$ é considerado um indicador limitado, uma medida-síntese do estado nutricional para análises populacionais, na medida em que a criança pode ser erroneamente classificada em "normal" para este indicador por expressar uma combinação de distúrbios nutricionais e não sua ausência. Por exemplo, crianças pequenas para idade, mas com peso ideal para idade pode mascarar uma desnutrição pregressa, já que o indicador $\mathrm{P} / \mathrm{I}$ indica desnutrição atual [16,29].

Já em um estudo do tipo caso-controle com crianças desnutridas (casos) e eutróficas (controles), que frequentavam duas Escolas Municipais de Educação Infantil, em São Paulo, foi verificado que tanto a IA familiar $\left(\mathrm{OR}=3,6\right.$; $\left.\mathrm{IC}_{95 \%}: 1,5-8,8\right)$ como o vínculo inadequado mãe-filho $\left(\mathrm{OR}=9,4\right.$; $\mathrm{IC}_{95 \%}$ : 3,7$24,0)$ estiveram associados com a desnutrição infantil $(p<0,05)^{[36] .}$
Essa associação pode ser resultado da proteção parental, onde os responsáveis pelas crianças pequenas, especialmente as mães, partilham recursos domésticos ou sacrificam seu próprio consumo de alimentos, protegendo as crianças de experimentarem fome, já que são extremamente dependentes dos

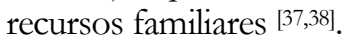

Pode-se deduzir, quanto à situação nutricional do grupo investigado, que algumas áreas do semiárido, mesmo com privilegiado IDHM, conseguem superar a desnutrição na sua forma aguda. No entanto, ainda é marcante a desnutrição pregressa, uma vez que o déficit estatural revela a história de atraso do crescimento linear ocorrido em algum momento da vida da criança por períodos anteriores de escassez alimentar ou processo infeccioso, desde o nascimento ou mesmo antes disso.

As associações contraditórias entre IA e desnutrição utilizando o IDHM como critério de seleção vêm sendo demonstradas em alguns estudos. Como a pesquisa realizada em Pelotas, cidade com alto IDHM, que encontrou baixa prevalência de IA (11\%) e mesmo assim altas prevalências de déficit em estatura (21\%) em crianças de 0 a 5 anos de famílias em IA, não condizendo com os achados para a região em pesquisas de porte nacional [39]. Já em uma pesquisa no segundo distrito de Duque de Caxias, o mais pobre do município do Rio de Janeiro, com elevadíssima prevalência de IA $(72 \%)$ foi averiguada na análise antropométrica que $8,6 \%$ das crianças de seis a trinta meses tinham déficit nutricional para A/I $[$ [ $]$.

\section{Excesso de peso}

No presente estudo, a prevalência de sobrepeso/obesidade (indicador $\mathrm{P} / \mathrm{A}=16,8 \%$ ) foi relevante, indicando progressão do quadro de transição nutricional, uma vez que no Ceará, em 2005, foi encontrado 6,5\% de menores de cinco anos com excesso de peso para o mesmo indicador [32]. Essa mudança tem sido relatada em muitas pesquisas, na qual suas causas primárias são referentes a fatores ambientais, principalmente a hábitos alimentares inadequados e ao sedentarismo, que contribuem para o acúmulo de gordura [10-12].

Essa situação já foi observada em famílias de Campinas (SP) em situação de IA, com dieta menos variada e não saudável [40,41], haja vista que famílias de baixa renda em IA sofrem tanto a diminuição da quantidade de alimentos como a perda da qualidade 
nutritiva. Já em níveis não tão graves de IA familiar, quando não envolve reduções na quantidade de ingestão de alimentos, a qualidade da dieta infantil pode ser comprometida, como o reduzido consumo de alimentos ricos em proteínas de alto valor biológico, além do consumo elevado de alimentos calóricos [42].

Em outro contexto, nos Estados Unidos da América (EUA), país desenvolvido com altas prevalências de excesso de peso na população, foi averiguado, a partir do National Health and Nutrition Examination Survey (NHANES) 1999-2002, que a IA é significativamente associada com risco de sobrepeso em crianças americanas de 3 a 5 anos de idade em famílias abaixo do nível de pobreza [43]. Sendo consistente com outra pesquisa implementada em Québec, de coorte de base populacional com crianças menores de cinco anos de idade, que encontrou uma associação positiva entre qualquer nível de insuficiência alimentar durante a infância ou período pré-escolar e sobrepeso [44].

Contudo, em estudo longitudinal americano, em 1998 e 1999, crianças com idade média de aproximadamente seis anos, de famílias com IA, foram $20 \%$ menos propensas a ter excesso de peso do que aquelas em segurança alimentar $(\phi=0,027)$, demonstrando no mesmo país a rápida transição da relação de IA com desnutrição para sobrepeso infantil [45].

Já em crianças de famílias de baixa renda que participavam de programa assistencial, em Massachusetts (EUA), evidenciou-se características peculiares em relação a IA e maiores chances de sobrepeso em meninas de 2 a 5 anos de idade $(O R=$ 1,47; $\left.\mathrm{IC}_{95 \%}: 1,05-2,06 ; p<0,05\right){ }^{[46]}$.

No Brasil, estudo realizado com beneficiários de programa de transferência de renda, Programa Bolsa Família, independentemente do grau de IA familiar, revelou maior inclusão de alimentos de maior teor energético e menor valor nutritivo, sendo fator de risco para excesso de peso na infância e adolescência [47].

\footnotetext{
Burlandy [48] comenta que famílias beneficiárias de programas de transferência condicionada de renda destinam, prioritariamente, os recursos recebidos com a compra de alimentos, principalmente para crianças. Mas é necessária a ação
}

conjunta com programas de nutrição para visualizar seu impacto sobre o estado nutricional; que, por sua vez, é influenciado por outros fatores como a disponibilidade e acesso a serviços de saúde; educação; saneamento; valor transferido; tamanho das famílias entre outros.

Quando se percebe as possíveis consequências do sobrepeso/obesidade em crianças, podemos considerar alarmante o que Kaiser \& Townsend [49] sugeriram, que crianças que crescem na pobreza são mais susceptíveis de se tornarem adultos obesos. Essa ideia pode ser reforçada pelas observações do NHANES III [50], em que meninas entre 2 e 7 anos de idade com insuficiência alimentar tiveram 1,6 vezes menos chances de ter excesso de peso. No entanto, meninas com idade entre 8 e 16 anos com alimentação insuficiente foram 3,5 vezes mais propensas a ter excesso de peso do que aquelas com alimentação suficiente.

Outra face da associação de pobreza, IA e sobrepeso em crianças e adolescentes foi relatada por Bhattacharya et al. [38]. Depois de analisar os dados do NHANES III, considerando raça/etnia, verificaram que a pobreza aumentou a incidência de sobrepeso, apenas entre as crianças hispânicas com idades entre 2 e 5 anos de idade como também para adolescentes hispânicos, entre 12 e 17 anos de idade.

Casey et al. [51] com objetivo de examinar e comparar as características das crianças americanas de familias em IA com consumo alimentar, inatividade física, baixo peso e excesso de peso, encontraram uma maior porcentagem de crianças com excesso de peso em famílias de baixa renda do que em famílias de renda mais alta, mostrando que a renda pode desempenhar papel maior do que a insuficiencia alimentar.

Logo, crianças de famílias pobres em situação de IA podem ser obesas devido a outros fatores associados a famílias de baixa renda. As possíveis razões para ligação entre obesidade e posição socioeconômica podem estar relacionadas à relação inversa de densidade energética da dieta e o custo monetário, resultando em dieta de baixa qualidade, ou seja, alimentos com excesso de calorias e pobres em nutrientes ${ }^{[52]}$.

Outra corrente de estudiosos defende que o aumento do risco do excesso de peso pode ser pela 
falta de um fornecimento estável de alimentos, levando a comer em excesso quando o alimento está disponível [53]. Além disso, de acordo com Rose \& Bodor [45], outros fatores são favoráveis para sobrepeso infantil, incluindo baixos níveis de atividade física, assistir televisão em excesso, etnia negra ou latina, elevado peso ao nascer e baixa renda. Assim, as atuais estratégias de prevenção da obesidade precisam ser revistas, uma vez que a obesidade ou sobrepeso infantil têm diversas variáveis associadas além dos fatores ambientais citados.

\section{Escala de percepção de Insegurança Alimentar e antropometria}

A hipótese inicial quanto aos indicadores de IA era referente à associação semelhante dos resultados entre a escala de percepção de IA e antropometria, que poderiam identificar o mesmo fenômeno, possibilitando classificar de maneira equivalente as familias em situação de IA. Na medida em que, segundo Pérez-Escamilla \& Segall-Corrêa [14], segurança alimentar e nutricional é o produto de vários fatores e da inter-relação entre eles, estando fortemente relacionado com a renda domiciliar per capita ${ }^{[54]}$.

Contudo, em nossos achados, a não relação significativa entre sobrepeso ou desnutrição com IA foi similar a outros estudos em municípios do semiárido brasileiro [33,34]. A situação sugere que $\mathrm{O}$ fenômeno de IA é mais dependente da vulnerabilidade econômica e social das famílias, e pode não guardar uma relação direta com a situação nutricional, que, por sua vez, também é mediado por outros fatores.

Além da interpretação complexa da associação da IA com situação nutricional, ao que se pode observar, há uma dificuldade de associação quando se analisa a IA com a situação nutricional, visto que os procedimentos metodológicos utilizados são de natureza distinta, sendo a escala de percepção de IA um método direto e a antropometria um método indireto. Além de que a antropometria é resultado da situação de saúde, servindo bem ao seu propósito de classificar o estado nutricional do indivíduo ${ }^{[55,56]}$.

Nesta perspectiva, a EBIA se comporta como um indicador de vulnerabilidade psicossocial, não apresentando a esperada associação entre a percepção de fome com déficit nutricional/excesso de peso, corroborando com as observações de outras pesquisas com crianças de localidades de generalizada pobreza no Brasil [33,34].

No entanto, na última década, ocorreram grandes avanços conceituais para a medida de SAN no Brasil, já que EBIA passou a ser um importante instrumento para a geração de indicador direto de medida domiciliar de segurança alimentar, sendo um método muito promissor para avaliar e monitorar efeitos das políticas públicas setoriais de combate à IA [57].

Vale destacar que os métodos de avaliação da IA se complementam e nenhum deles deve ser considerado superior em relação a outro. Na maioria dos casos, o método de escolha depende da questão que precisa ser atendida, bem como o orçamento disponível para realizar a avaliação ${ }^{[14]}$.

\section{CONCLUSÕES}

A prevalência de sobrepeso encontrada demonstra a necessidade de interferência de políticas públicas emergenciais, haja vista que a obesidade na infância tende a se manter na idade adulta, levando à consequências catastróficas para a economia do país e para a saúde pública, por ser causa de inúmeras doenças crônicas de alta morbimortalidade, aumentando custos no setor privado e público e diminuindo drasticamente a sobrevida do indivíduo e, por consequência, da população.

Neste estudo não foi encontrada uma situação nutricional determinante para crianças menores de cinco anos e IA. Diante disso, os pontos de interações entre IA e situação nutricional se comportam de maneira divergente da expectativa esperada, talvez reflexo do processo de transição nutricional que se comporta de maneira particular nas diversas realidades.

Pode-se aceitar que a não associação entre a escala de percepção de IA (EBIA) e antropometria são indicadores que medem, apropriadamente, fenômenos distintos, haja vista que os resultados das pesquisas anteriores apresentam consistência entre si e guardam correspondência muito forte com os atuais achados.

Dessa maneira, os esforços para diminuir a IA e obesidade infantil funcionariam de formas independentes. Entretanto, propiciar o 
desenvolvimento de intervenções multiprofissionais relacionadas com políticas de SAN poderão trazer benefícios para compreensão de suas dimensões, uma vez que seu conceito transpassa as muitas disciplinas e campos de saberes.

\section{AGRADECIMENTOS}

Agradecemos à Secretaria de Saúde Municipal do Crato (CE); ao Laboratório de Segurança Alimentar e Nutricional da Universidade Estadual do Ceará (UECE); aos acadêmicos voluntários da Universidade Regional do Cariri; à Coordenação de Aperfeiçoamento de Pessoal de Nível Superior (CAPES), pela bolsa de estudo, vinculada ao Programa de Pós-graduação em Ciências da Nutrição da Universidade Federal da Paraíba (UFPB).

\section{REFERÊNCIAS}

[1] Brasil. Ministério da Saúde. Conselho de Segurança Alimentar e Nutricional. Princípios e diretrizes de uma política de segurança alimentar e nutricional. In: Conferência Nacional de Segurança Alimentar e Nutricional. Brasília: CONSEA; 2004.

[2] Brasil. Lei no 11.346 , de 15 de setembro de 2006. Cria o Sistema Nacional de Segurança Alimentar e Nutricional (SISAN) com vistas em assegurar o direito humano à alimentação e dá outras providências. Diário Oficial da União, Brasília, 18 set. 2006. Seção 1, p. 1.

[3] Prado SD, Gugelmin SA, Mattos RA, Silva JK, Olivares PSG. A pesquisa sobre segurança alimentar e nutricional no Brasil de 2000 a 2005: tendências e desafios. Ciênc Saúde Colet. 2010;15(1):7-18.

[4] Castro LMC. Pesquisar sobre segurança alimentar e nutricional no Brasil: a que viemos? Ciênc Saúde Colet. 2010;15(1):26-28.

[5] Instituto Brasileiro de Geografia e Estatística. Pesquisa Nacional por Amostra de Domicílios - PNAD: Segurança Alimentar 2004/2009. Rio de Janeiro: IBGE; 2010.

[6] Barroso GS, Sichieri R, Salles-Costa R. Fatores associados ao déficit nutricional em crianças residentes em uma área de prevalência elevada de insegurança alimentar. Rev Bras Epidemiol. 2008;11(3):484-94.

[7] Batista Filho M. Introdução. Cad Estud Desenv Soc Debate. 2006;4:9-16.
[8] Batista Filho M, Rissin A. A transição nutricional no Brasil: tendências regionais e temporais. Cad Saúde Pública. 2003;19(Suppl 1):S181-91.

[9] Instituto Brasileiro de Geografia e Estatística. Pesquisa de Orçamentos Familiares - POF 2008-2009. Antropometria e estado nutricional de crianças, adolescentes e adultos no Brasil. Rio de Janeiro: IBGE, 2010.

[10] Caballero B. Subnutrição e obesidade em países em desenvolvimento. Cad Estud Desenv Soc Debate. 2005;2:10-13.

[11] Doak CM, Adair LS, Bentley M, Monteiro C, Popkin BM. The dual burden household and the nutrition transition paradox. Int J Obes. 2005;29(1):129-36.

[12] Sawaya AL, Solymos GMB, Florêncio TMMT, Martins PA. Os dois Brasis: quem são, onde estão e como vivem os pobres brasileiros? Estud Av. 2003;17(48):21-44.

[13] Food and Agriculture Organization. Measurement and assessment of food deprivation and undernutrition. Rome: FAO; 2003.

[14] Pérez-Escamilla R, Segall-Corrêa AM. Food insecurity measurement and indicators. Rev Nutr. 2008;21(Suppl.):15s26s.

[15] Segall-Corrêa AM. Insegurança alimentar medida a partir da percepção das pessoas. Estud Av. 2007;21(60):14354.

[16] Vasconcelos FAG. Avaliação nutricional de coletividades. Florianópolis: UFSC; 2007.

[17] Teixeira-Lima R. Condições de nascimento e desigualdade social [tese]. São Paulo: Faculdade de Saúde Pública, Universidade de São Paulo; 2001. 187 p.

[18] Programas das Nações Unidas para o Desenvolvimento. Atlas do Desenvolvimento Humano no Brasil. Brasília: PNUD; 2003.

[19] Instituto Brasileiro de Geografia e Estatística. IBGE cidades@. 2010. [acesso em 15 maio 2010]. Disponível em: http://www.ibge.gov.br/cidadesat/painel/painel.php?codm $\underline{\text { un }=230420}$

[20] Fundação Cearense de Meteorologia e Recursos Hídricos. Zoneamento geoambiental do Estado do Ceará: parte II mesorregião do sul cearense. Fortaleza: FUNCEME; 2006. 
[21] Brasil. Sistema de Informação do Programa Nacional de Imunizações. DATASUS. Campanha nacional de vacinação contra pólio 2009, Ceará. [acesso em 10 maio 2010]. Disponível

em: http://pni.datasus.gov.br/Consulta Polio2 Mun 09.asp?U $\underline{\mathrm{F}=23 \& \text { faixas }=\text { todas }}$

[22] Instituto Brasileiro de Geografia e Estatística. Pesquisa Nacional por Amostra de Domicílios - PNAD: suplemento sobre segurança alimentar 2004. Rio de Janeiro: IBGE; 2006.

[23] Associação Brasileira de Empresas de Pesquisa. Critério de Classificação Econômica Brasil. São Paulo: ABEP; 2010. [acesso em 28 maio 2010]. Disponível em: http://www.abep.org/novo/Content.aspx?ContentID=301

[24] Brasil. Ministério da Saúde. CGPAN. Vigilância alimentar e nutricional - SISVAN: orientações básicas para a coleta, processamento, análise de dados e informação em serviços de saúde. Norma técnica - SISVAN. Brasília: Ministério da Saúde; 2008.

[25] World Health Organization.WHO Anthro for personal computers: Software for assessing growth and development of the world's children [computer program], version 3.1, 2010. Geneva: WHO; 2010.

[26] World Health Organization. WHO Multicentre Growth Reference Study Group. WHO child growth standards: length/height-for-age, weight-for-age, weight-for-length, weight-for-height and body mass index-for-age: methods and development. Geneva: WHO; 2006.

[27] Segall-Corrêa AM, Pérez-Escamilla R, Maranha LK, Sampaio MFA, Yuyama L, Alencar F, et al. Projeto: acompanhamento e avaliação da segurança alimentar de famílias brasileiras: validação de metodologia e de instrumento de coleta de informação. Campinas: Departamento de Medicina Preventiva e Social, UNICAP/ OPAS/Ministério da Saúde; 2004. (Relatório Técnico).

[28] Brasil. Ministério da Saúde. Conselho Nacional de Saúde, Resolução 196, de 10 de outubro de 1996. Aprova diretrizes e normas regulamentadoras de pesquisas envolvendo seres humano. Diário Oficial da União, Brasília, 16 out. 1996. Seção 1, p. 21082.

[29] Brasil. Ministério da Saúde. Pesquisa Nacional de Demografia e Saúde da Criança e da Mulher - PNDS 2006: dimensões do processo reprodutivo e da saúde da criança. Brasilia: Ministério da Saúde; 2009.

[30] Monteiro CA, Benicio MHD, Konno SC, Silva ACF, Lima ALL, Conde WL. Causas do declínio da desnutrição infantil no Brasil, 1996-2007. Rev Saúde Públ. 2009;43(1):3543.

[31] Monteiro CA, Conde WL, Konno SC. Análise do inquérito chamada nutricional 2005. Cad Estud Desenv Soc Debate. 2006;4:29-36.

[32] Diniz DB, Fernandes DLA, Mamede ALES. Estado nutricional de crianças menores de cinco anos do semiárido do Ceará. Cad Estud Desenv Soc Debate. 2006;4:51-6.

[33] Oliveira JS, Lira PIC, Andrade SLLS, Sales AC, Maia SR, Batista Filho MB. Insegurança Alimentar e estado nutricional de crianças de São João do Tigre, no semiárido do Nordeste. Rev Bras Epidemiol. 2009;12(3):413-23.

[34] Oliveira JS, Lira PIC, Maia SR, Siqueira LAS, Amorim RCA, Batista Filho MB. Insegurança alimentar e estado nutricional de crianças de Gameleira, zona da mata do Nordeste brasileiro. Rev Bras Saude Mater Infant. 2010;10(2):237-45.

[35] Isanaka S, Mora-Plazas M, Lopez-Arana S, Baylin A, Villamor E. Food Insecurity Is Highly Prevalent and Predicts Underweight but Not Overweight in Adults and School Children from Bogota, Colombia. J Nutr. 2007;137(12):2747-55.

[36] Vieira VL, Souza MP, Cervato-Mancuso AM. Insegurança alimentar, vínculo mãe-filho e desnutrição infantil em área de alta vulnerabilidade social. Rev Bras Saude Mater Infant. 2010;10(2):199-207.

[37] Parker L. Obesity, food insecurity and the federal child nutrition programs: Understanding the linkages. Food Research and Action Center, 2005 [cited 2012 Nov 03]. Available from: http://www.rwif.org/en/researchpublications/find-rwjf-research/2005/10/obesity--foodinsecurity-and-the-federal-child-nutrition-program.html

[38] Bhattacharya J, Currie J, Haider S. Poverty, food insecurity, and nutritional outcomes in children and adults. J Health Econ. 2004;23(4):839-62.

[39] Santos JV, Gigante DP, Domingues MR. Prevalência de segurança alimentar em Pelotas, Rio Grande do Sul, Brasil, e estado nutricional de indivíduos que vivem nessa condição. Cad Saúde Pública. 2010;26(1):41-9.

[40] Marín-León L, Segal-Corrêa AM, Panigassi G, Maranha LK, Sampaio MFA, Pérez-Escamilla R. A percepção de insegurança alimentar em famílias com idosos em Campinas, São Paulo, Brasil. Cad Saúde Pública. 2005;21(5):1433-40. 
[41] Panigassi G, Segal-Corrêa AM, Marín-León L, PérezEscamilla R, Maranha LK, Sampaio MFA. Insegurança alimentar intrafamiliar e perfil de consumo de alimentos. Rev Nutr. 2008;21(Suppl):S135-44.

[42] Antunes MML, Sichieri R, Salles-Costa R. Consumo alimentar de crianças menores de três anos residentes em área de alta prevalência de insegurança alimentar domiciliar. Cad Saúde Pública. 2010;26(8):1642-50.

[43] Casey PH, Simpson PM, Gossett JM, Bogle ML, Champagne CM, Connell C, et al. The Association of Child and Household Food Insecurity With Childhood Overweight Status. Pediatrics. 2006;118(5):e1406-e13.

[44] Dubois L, Farmer A, Girard M, Porcherie M. Family food insufficiency is related to overweight among preschoolers'. Soc Sci Med. 2006;63(6):1503-16.

[45] Rose D, Bodor N. Household food insecurity and overweight status in young school children: Results from the early childhood longitudinal study. Pediatrics. 2006;117(2):464-73.

[46] Metallinos-Katsaras E, Sherry B, Kallio J. Food Insecurity Is Associated with Overweight in Children Younger than 5 Years of Age. J Am Diet Assoc. 2009;109(10):1790-4.

[47] Segall-Corrêa AM, Salles-Costa R. Novas possibilidades de alimentação a caminho? Democracia Viva. 2008;39:6873 .

[48] Burlandy L. Transferência condicionada de renda e segurança alimentar e nutricional. Ciênc Saúde Colet. 2007;12(6):1441-51.
[49] Kaiser LL, Townsend MS. Food insecurity among US children. Top Clin Nutr. 2005;20(4):313-20.

[50] Alaimo K, Olson CM, Frongillo EA. Low family income and food insufficiency in relation to overweight in US children. Arch Pediatr Adolesc Med. 2001;155(10):116167.

[51] Casey PH, Szeto K, Lensing S, Bogle M, Weber J. Children in food insufficient, low-income families. Arch Pediatr Adolesc Med. 2001;155(4):508-14.

[52] Drewnowski A, Darmon N. The economics of obesity: Dietary energy density and energy cost. Am J Clin Nutr. 2005; 82(Suppl.):265S-73S.

[53] Kaiser LL, Melgar-Quiñonez HR, Lamp CL, Johns MC, Sutherlin JM, Harwood JO. Food security and nutritional outcomes of preschool-age Mexican-American children. J Am Diet Assoc. 2002;102(7):924-29.

[54] Hoffmann R. Determinantes da insegurança alimentar no Brasil. Análise dos dados da PNAD de 2004. Seg Alim Nutr. 2008;15(1):49-61.

[55] Townsend MS, Peerson J, Love B, Achterberg C, Murphy SP. Food insecurity is positively related to overweight in women. J Nutr. 2001;131(6):1738-45.

[56] Drewnowski A, Specter SE. Poverty and obesity: the role of energy density and energy costs. Am J Clin Nutr. 2004;79(1):6-16.

[57] Segall-Corrêa AM, Marin L. A segurança alimentar no Brasil: proposição e usos da escala brasileira de medida da insegurança alimentar (EBIA) de 2003 a 2009. Seg Alim Nutr. 2009;16(2):1-19. 